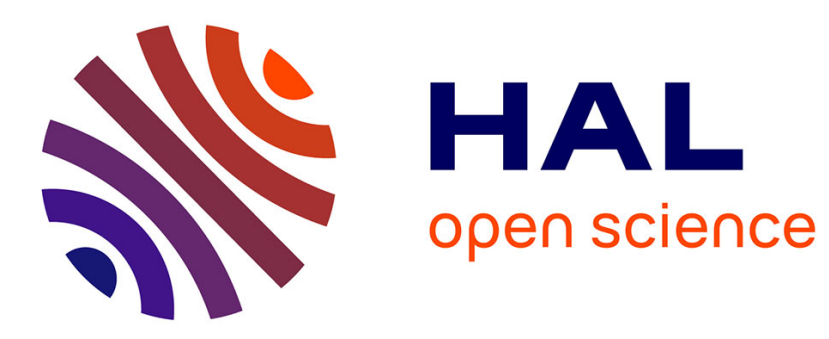

\title{
L'industrie d'armement de la Russie
}

\author{
Jacques Fontanel, Alexandre Karlik
}

\section{To cite this version:}

Jacques Fontanel, Alexandre Karlik. L'industrie d'armement de la Russie. Guerres et conflits économiques, Office des Publications Universitaires, 2005, 9901.0.0877.4. hal-03202098

\section{HAL Id: hal-03202098 \\ https://hal.univ-grenoble-alpes.fr/hal-03202098}

Submitted on 26 Apr 2021

HAL is a multi-disciplinary open access archive for the deposit and dissemination of scientific research documents, whether they are published or not. The documents may come from teaching and research institutions in France or abroad, or from public or private research centers.
L'archive ouverte pluridisciplinaire HAL, est destinée au dépôt et à la diffusion de documents scientifiques de niveau recherche, publiés ou non, émanant des établissements d'enseignement et de recherche français ou étrangers, des laboratoires publics ou privés. 


\title{
L'industrie d'armement de la Russie
}

\author{
Fontanel Jacques, Alexandre Karlik
}

in

Guerres et conflits économiques (Jacques Fontanel)

Office des Publications Universitaires,

Alger, 2005.

Résumé: Le complexe militaro-industriel soviétique a subi une crise économique profonde après l'émergence de l'économie de la transition et la fin de la course aux armements dans le monde. Les dépenses militaires ont considérablement été réduites en Russie et la crise a touché principalement l'industrie d'armement. Si l'armement nucléaire reste bien l'instrument essentiel de la défense russe, les demandes d'équipement de l'armée ruse n'ont que rarement été satisfaites. Les entreprises ont été obligées de conduire des stratégies de diversification de la production d'armement, d'exporter les armes que le Ministère de la défense ne leur commandait plus, de s'engager dans l'aide technique auprès des pays intéressés et de convertir leurs activités vers des productions civiles. Le secteur militaire est dépendant du niveau de développement économique, des capacités nationales disponibles et de la recherche)développement, autant de facteurs qui ne présagent guère d'un renouveau rapide de la puissance militaire russe.

The Soviet military-industrial complex suffered a deep economic crisis after the emergence of the transition economy and the end of the global arms race. Military spending in Russia has been drastically reduced and the crisis has mainly affected the arms industry. While nuclear weapons remain the main instrument of Russian defence, the demands of the Russian military for equipment have rarely been met. Companies have been forced to pursue strategies of diversification of arms production, exporting weapons that the Ministry of Defence no longer orders, engaging in technical assistance to interested countries and converting their activities to civilian production. The military sector is dependent on the level of economic development, available national capabilities and research and development, all of which do not bode well for a rapid renewal of Russian military power.

Russian military-industrial complex, Russian military expenditures, conversion of the military sector, arms exports

Complexe militaro-industriel russe, exportations d'armes, conversion du secteur militaire, dépenses militaires russes 
La sécurité d'un pays n'est jamais gratuite. Elle implique des coûts, qui sont justifiés par l'importance des menaces concernant la sécurité internationale. En ce sens, une industrie nationale d'armement n'a de sens qu'au regard des menaces militaires présentes et futures d'un pays. Autrement dit, son poids et son évolution dépendent à la fois de celles des pays adversaires potentiels et de celles de ses alliés. Selon son importance, ses alliances et les menaces, un pays s'engage dans la production d'armes, en vue de bénéficier d'une indépendance dans la disponibilité et l'utilisation des matériels de défense. L'industrie d'armement de la Russie ne peut se mesurer qu'à l'aulne de la puissance américaine, de l'OTAN et de la Chine.

Le développement de la production d'armement dépend des choix des gouvernements, lesquels sont la résultante de considérations stratégiques, politiques et économiques. D'abord, l'évolution des dépenses militaires des ennemis potentiels est un facteur explicatif de la course aux armements. Aujourd'hui, la Russie ne connait pas nécessairement ses ennemis ${ }^{1}$. Sauf à souhaiter redevenir une puissance alternative aux Etats-Unis, la Russie n'a pas de raison conflictuelle à augmenter considérablement ses dépenses militaires. Elle doit le faire pour sa propre sécurité intérieure et pour compenser l'obsolescence 'et le vieillissement de ses matériels. Elle doit aussi faire face aux nouvelles stratégies militaires, qui impliquent de nouveaux moyens d'action et d'armes.

L'industrie d'armement et l'équipement de l'armée russe ont subi de plein fouet l'effondrement des dépenses militaires de la Russie $^{2}$. Le complexe militaro-industriel a été amené à engager une

\footnotetext{
${ }^{1}$ La Chine reste toujours une menace, les Etats-Unis sont devenus une hyper puissance, l'Europe devient un partenaire économique souhaité.

${ }^{2}$ Fontanel J., Coulomb F., Samson I., (2001), « Military conversion and transition in Russia ", Pax Economica, $n^{\circ}$ G, 2001. Fontanel J., Borisova I., Ward M., (1995), "The principles of arms conversion in the case of Russia », Defence and Peace Economics,
$1995,6.3$.
} 
politique de diversification vers les produits civils et l'exportation. L'industrie d'armement elle-même a été doublement dépendante des commandes de l'armée et des ventes à l'étranger.

\section{I. - Les dépenses militaires de la Russie}

Il y a quelques années les comparaisons des dépenses militaires faisaient l'objet de débats. Au début des années 1980, le SIPRI considérait que l'URSS dépensait un bon tiers de milliards de dollars de moins que les Etats-Unis à des fins militaires, l'USACDA estimait, au contraire que l'Union soviétique dépensait un bon tiers de plus. Le fardeau militaire a constitué l'une des raisons de l'effondrement de l'URSS, sans doute d'ailleurs la plus importante. Aujourd'hui, les Etats-Unis dépensent $40 \%$ des dépenses militaires mondiales, soit autant que l'URSS et les pays européens dans leur ensemble.

Les dépenses militaires de la Russie ont connu une très forte baisse après l'engagement du processus de la transition.

Tableau 1

Le budget de la défense russe de 1992 à 2001 (en milliards de roubles)

\begin{tabular}{|c|c|c|c|c|}
\hline Années & Budget initial & Budget révisé & Budget final & $\begin{array}{l}\text { En indices constants } \\
\text { (base 1992) }\end{array}$ \\
\hline 1992 & 0,38 & & 0,86 & 100 \\
\hline 1993 & 3,12 & 8,33 & 7,21 & 85 \\
\hline 1994 & 40,63 & & 28,03 & 77 \\
\hline 1995 & 48,58 & 59,38 & 47,80 & 43 \\
\hline 1996 & 80,19 & & 63,9 & 25 \\
\hline 1997 & 104,30 & 83,00 & 79,70 & 27 \\
\hline 1998 & 81,77 & & 65,10 & 18 \\
\hline 1999 & 93,70 & 109,00 & 116,80 & 26 \\
\hline 2000 & 143,00 & & 190,8 & 35 \\
\hline 2001 & 218,92 & & 240,0 & 37 \\
\hline 2002 & 262,90 & & & \\
\hline
\end{tabular}

Aujourd'hui, au taux de change officiel, les dépenses militaires de la Russie n'atteignent pas 8 milliards de dollars. Avec le système des parités de pouvoir d'achat, cette somme peut être multipliée par un facteur quatre. Il n'en reste pas moins vaai que la Russie ne dépense qu'à peine $10 \%$ des dépenses militaires américaines. On constate que les dépenses militaires de la Russie représentent un fardeau de moins en moins important eu égard à la richesse produite chaque année par 
le pays. Ces résultats sont proches de ceux de la France. Les dépenses dites de coûts opérationnels représentent près des plus de $60 \%$ des dépenses totales, alors même que plus d'un tiers des sommes engagées est inscrit sous le libellé "Autres». Notons que les subventions en faveur des industries de défense représentent moins d'un demi-milliard de roubles, soit $0,15 \%$ des dépenses totales.

Au regard de la richesse produite annuellement, les dépenses militaires officielles connaissent une réduction de grande importance, même si ce trend s'est renversé ces trois dernières années. Le Ministre de la défense Sergei Ivanov considère que $70 \%$ du budget est dépensé pour les troupes et les bureaucrates, ce qui laisse trop peu de moyens pour maintenir et améliorer les équipements ${ }^{3}$. En janvier 2000, le gouvernement a décidé d'acheter $50 \%$ d'équipement supplémentaire, ce qui n'a d'ailleurs pas amélioré substantiellement la situation. Malgré tous les efforts financiers, les militaires russes travaillent toujours très majoritairement avec des armes de plus de 10 ans, souvent obsolètes.

La Russie a décroché en termes de puissance militaire. Il lui reste l'effet " patrimoine ", lequel lui donne plus de force réelle que l'énoncé de ses flux ne semble l'indiquer, même si le patrimoine se doit d'être maintenu. Il est impossible à la Russie de dépenser plus de 200 milliards de dollars à des fins militaires pour espérer seulement rester compétitive. En fait, ce "gap» peut conduire la Russie à chercher d'autres alliances peut-être avec la Chine.

\footnotetext{
3 Wines M., (2000), "Putin cuts forces by 600 000, Promising Military Overhaul »,
} New York Times, November. 10. 
Tableau 2

Le budget officiel de la défense russe en 2001, en milliards de roubles

\begin{tabular}{|c|c|}
\hline Lignes du budget militaire & Dépenses \\
\hline $\begin{array}{l}\text { Maintenance Personnel } \\
\text { - Allocations (y compris le personnel civil) } \\
\text { - Alimentation } \\
\text { - Congés et repos médicaux } \\
\text { - Bénéfices et compensations }\end{array}$ & $\begin{array}{r}62,5 \\
17,0 \\
3,6 \\
1,4\end{array}$ \\
\hline $\begin{array}{l}\text { Entraînement au combat et logistique } \\
\text { - Maintenance des immeubles et réparations } \\
\text { - Stocks et produits énergétiques spéciaux } \\
\text { - Transports } \\
\text { - Maintenance, opérations et réparations des propriétés et installations } \\
\text { - Autres }\end{array}$ & $\begin{array}{r}15,9 \\
12,0 \\
5,7 \\
1,8 \\
1,9\end{array}$ \\
\hline $\begin{array}{l}\text { Programme énergie atomique } \\
\text { Mobilisation et entraînement des réserves } \\
\text { Sécurité collective de la CEI et maintien de la paix } \\
\text { Dépenses d'éducation et de santé } \\
\text { Garanties d'assurance } \\
\text { Commande militaire centrale } \\
\text { Industries de défense } \\
\text { Autres }\end{array}$ & $\begin{array}{l}5,1 \\
2,3 \\
2,7 \\
2,2 \\
1,5 \\
0,9 \\
0,3 \\
74,8\end{array}$ \\
\hline TOTAL & \\
\hline
\end{tabular}

Tableau 3

L'importance des dépenses militaires au regard du PIB (selon les sources officielles)

\begin{tabular}{|c|c|}
\hline Années & $\%$ des dépenses militaires/PIB \\
\hline 1992 & 4,7 \\
\hline 1993 & 4,4 \\
\hline 1994 & 4,6 \\
\hline 1995 & 3,1 \\
\hline 1996 & 3,0 \\
\hline 1997 & 3,1 \\
\hline 1998 & 2,4 \\
\hline 1999 & 2,6 \\
\hline 2000 & 2,7 \\
\hline 2001 & 2,8 \\
\hline
\end{tabular}




\section{II. - L'importance économique cu complexe militaro-industriel russe}

Avec le système centralisé de l'URSS, le complexe militaro-industriel était considéré comme le fer de lance de l'économie nationale. Il produisait $100 \%$ des magnétoscopes, $94 \%$ des télévisions, $55 \%$ des aspirateurs. Pris en son sens large, il comprenait 20 millions de personnes. À partir de 1991, la conversion est devenue une nécessité économique du fait de la brutale et forte contraction des crédits militaires. La thérapie de choc a sérieusement ébranlé les certitudes et la puissance du CMI. Les entreprises ont été amenées à engager des stratégies de diversification de la production d'armements et à se réorganiser en faveur de la production civile, dans le cadre d'un marché. Le bilan, dix années plus tard, n'est pas réjouissant. Pour quelques cas de conversions réussies, la plupart des entreprises d'armement connaissent de graves difficultés économiques ${ }^{4}$.

Aujourd'hui, le complexe militaro-industriel ne produit pas que des armements. Dans ces conditions, il faut distinguer son état économique de celui de la production spécifique d'armement. Fin 2003, le registre des entreprises du complexe industriel de défense (CMI) comprenait 1279 entreprises. En 2002, dans les huit industries militaires, on comptait environ 2000 entreprises et organisations. Ces entreprises sont situées dans 72 régions de la Fédération de Russie. Une part importante de la production industrielle et scientifique du CMI est normalement destinée à satisfaire les besoins fédéraux. Elle est donc financée par le budget fédéral. En 2004, un financement de plus de 330 milliards de roubles du budget fédéral est prévu (soit 10 milliards de dollars). Du fait du caractère limité de la privatisation dans le secteur, la participation d'Etat dans la structure de la propriété reste élevée 5 .

Le CMI assure environ $25 \%$ de la production de construction mécanique, et plus de 40\% des exportations de la branche. Au sein du CMI, les industries les plus tournées vers les exportations (les deuxtiers' de la production) sone l'aéronautique, la construction navale et radio industrie. Les exportations totales d'armes prévues pour 2003

${ }^{4}$ Fontanel J., Coulomb F., Samson I., (2001), « Military conversion and transition in Russia ", Pax Economica, n6, 2001. Fontanel J., Borisova I., Ward M., (1995), «The principles of arms conversion in the case of Russia ", Defence and Peace Economics, 1995, 6.3.

${ }^{5}$ Au $1^{\text {er }}$ juin 2003 en Russie, on compte 9860 entreprises fédérales unitaires. De plus, l'Etat détient les actions de 4205 sociétés anonymes. 
s'élèvent à 5,6 milliards de dollars. (4,5 milliards de dollars en 2002). Aujourd'hui, la Russie est le troisième exportateur mondial d'armes, derrière les Etats-Unis et la Grande-Bretagne.

Les industries du CMI absorbent 25 à 30\% environ des investissements en capital. Les tendances de l'état de l'appareil productif des entreprises du CMI correspondent à des tendances générales dans l'industrie. L'usure et l'obsolescence des fonds de production actifs est de l'ordre de $70-80 \%$. La part des équipements à durée d'utilisation inférieure à 5 ans ne représente que $4,5 \%$ (contre $5,7 \%$ en moyenne dans l'industrie russe). La part des équipements, ayant servi depuis plus de 20 ans, est de $32 \%$ ( $41,5 \%$ en moyenne dans l'industrie). Or, pour le bon fonctionnement des processus tech-niques de la production des armements, la part des équipements à durée d'utilisation de moins de 10 ans doit être supérieure à $70 \%$, alors qu'en réalité, cet indicateur s'élève à $28 \%(15,3 \%$ en moyenne dans l'industrie). Cette situation résulte de l'insuffisance du taux de rénovation de l'appareil productif dans l'industrie. Le taux d'utilisation des capacités de production atteint $30-40 \%$ dans le CMI, ce qui est inférieur à la moyenne $(51 \%)$ dans la construction mécanique.

Selon les chiffres officiels de la Russie, entre 1991 et 1995, 2,5 millions de personnes ont quitté les entreprises du CMI. Il en restait 3,6 millions, utilisés à peine à $10 \%$ de leur potentiel ${ }^{6}$. Aujourd'hui, environ 2 millions de personnes sont employées dans le CMI, la majorité étant concentrée dans l'aéronautique, l'industrie spatiale et l'industrie des armements. La chute de la production, dans le contexte de retard considérable de salaires par rapport à la moyenne nationale, a affaibli considérablement la qualité des ressources humaines potentielles du CMI. Les résultats de l'enquête de début 2001, effectuée auprès des directeurs d'entreprises de défense, ont montré que moins de $40 \%$ de salariés seulement répondaient aux exigences du travail dans les conditions du marché. Les collèges techniques professionnels et les établissements d'enseignement professionnel supérieur et secondaire ont pratiquement arrêté la préparation du personnel qualifié spécialisé dans l'armement ${ }^{7}$.

"Sergounin A.A., Subbotin S.V., (1999), "Russian arms transfers to East Asia in the 1990 s ", SIPRI Research Report n¹5, Oxford University Press, New York, pp.15-16.

${ }^{7}$ En 1996-2001, l'âge moyen des salariés des entreprises du CMI a augmenté considérablement (de 47 à 58 ans). Dans les organisations de recherche et de conception du CMI, l'âge moyen des salariés est encore plus élevé. Or, d'après les estimations, la part des salariés de plus de 50 ans ne doit pas dépasser $20 \%$, la sécurité économique de la production étant mise en question dans le cas contraire. 
La structure du système d'approvisionnement technique et en matériaux des entreprises du CMI, très complexe, comprend 30000 milliers de fournisseurs. Cutre le degré élevé de la coopération intra et interbranche, les entreprises du CMI se caractérisent par une certaine dépendance à l'égard des importations de composants. L'éclatement de l'URSS en 1991 a mis la fin à l'existence du CME unique, basée sur la localisation planifiée des unités de production de l'industrie de défense sur l'ensemble du territoire de l'Union soviétique. La Russie a hérité environ $80 \%$ du potentiel de défense de l'URSS, mais par ses propres capacités, elle ne peut assurer que $17 \%$ de la production du CMI de l'URSS. Bien que la production de certains composants pour le CMI soit organisée sur le territoire de la Russie, la dépendance à l'égard de la coopération avec les entreprises des pays de la CEI, notamment, de la Biélorussie, l'Ukraine et le Kazakhstan, persiste.

Le CMI de la Russie connaît une baisse continue de la production, accompagnée d'une modification de la structure de production vers l'augmentation de la production à usage civil. Elle dispose de capacités excessives dans la production militaire, et, en même temps, elle subit une sous-capacité en termes de potentiel d'innovation (tableau 4). Elle subit la diminution de son niveau d'investissements et, par conséquent, du taux de rénovation des fonds de production fixe, elle doit faire face à l'usure et l'obsolescence rapides de l'appareil de production et à la détérioration de la structure technologique.

Le CMI souffre du bas niveau d'emploi du personnel et d'un niveau de salaire relativement faible, étant donné de fortes exigences de qualification. La situation économique et financière de nombre d'entreprises et d'organisations s'avère très difficile. Les dettes du ministère de la Défense russe pour des commandes d'Etat déjà réalisées dans le domaine des armes atteignent 20 milliards de roubles. À titre d'exemple, le besoin annuel en fonds pour le maintien des capacités de mobilisation représente 4 à 5 milliards de roubles. Or, en 1995, 200 millions de roubles avaient été prévus. 


\section{Tableau 4}

L'état des capacités de production dans l'industrie russe (d'aptès les enquêtes trimestrielles des directeurs d'entreprises industrielles)

\begin{tabular}{|l|c|c|}
\hline Industries & $\begin{array}{l}\text { La part des capacités } \\
\text { de production exis- } \\
\text { tantes, pouvant être } \\
\text { utilisées en cas de } \\
\text { croissance de la } \\
\text { demande de la } \\
\text { production } \\
\text { traditionnelle, } \%\end{array}$ & $\begin{array}{l}\text { La part des capacités } \\
\text { de production } \\
\text { existantes, pouvant } \\
\text { être utilisée en cas de } \\
\text { croissance d'une } \\
\text { nouvelle demande, } \\
\%\end{array}$ \\
\hline Industrie, total & 10 & 4 \\
\hline Combustibles & 5 & 1 \\
\hline Métaux ferreux & 14 & 3 \\
\hline Métaux non ferreux & 3 & 2 \\
\hline Industrie chimique et pétrochimique & 11 & 3 \\
\hline $\begin{array}{l}\text { Construction mécanique et industrie } \\
\text { des métaux }\end{array}$ & 10 & 7 \\
\hline $\begin{array}{l}\text { Industrie du bois, ébénisterie, } \\
\text { industrie du papier et de la pâte à } \\
\text { papier }\end{array}$ & 8 & 3 \\
\hline $\begin{array}{l}\text { Industrie des matériaux de } \\
\text { construction }\end{array}$ & 10 & 6 \\
\hline Industrie légère & 13 & 4 \\
\hline Industrie alimentaire & 18 & 3 \\
\hline
\end{tabular}

Tableau 5

La structure d'âge de l'équipement de production dans l'industrie (en $\%$ du total) ${ }^{8}$

\begin{tabular}{|c|c|c|c|c|c|}
\hline Années & \multicolumn{4}{|c|}{ Age } & Age moyen, ans \\
\hline & $\begin{array}{l}\text { Móins } \\
\text { de } 5 \text { ans }\end{array}$ & $6-10$ ans & $11-20$ ans & $\begin{array}{c}\text { Plus } \\
\text { de } 20 \text { ans }\end{array}$ & $\begin{array}{c}\text { Age moyen } \\
\text { de l'équipement, ans }\end{array}$ \\
\hline 1970 & 40,6 & 30,2 & 21,0 & 8,2 & 8,4 \\
\hline 1975 & 37,5 & 29,7 & 23,8 & 9,0 & 8,9 \\
\hline 1980 & 35,5 & 28,8 & 25,1 & 9,6 & 9,5 \\
\hline 1985 & 33,1 & 28,2 & 25,8 & 12,9 & 10,1 \\
\hline 1990 & 29,4 & 28,3 & 27,3 & 15,0 & 10,8 \\
\hline 1995 & 10,1 & 29,8 & 36,7 & 23,2 & 14,3 \\
\hline 2000 & 4,7 & 10,6 & 46,5 & 38,2 & 18,7 \\
\hline 2001 & 5,7 & 7,6 & 45,2 & 41,5 & 19,4 \\
\hline
\end{tabular}

8 Source : Osnovnye fondy i drugie nefinansovie aktivy v Rossii, 1999, s. 27. Statisticheskiy bulleten, $\mathrm{N}^{\circ} 9$ (93), ianvar' 2003. (Fonds fixes et d'autres actifs non financiers en Russie, 1999, p 27. Le bulletin statistique, $N^{\circ} 9$ (93), janvier 2003). 
Puisque la part de la production réalisée dans le cadre de la commande de défense d'Etat est faible, les entreprises du CMI s'orientent essentiellement vers l'exportation des armements, et vers la production civile destinée au marché interne ( $42 \%$ environ), dont presque $80 \%$ est constituée par la production à usage industriel. Les directions principales de la politique publique à l'égard du CMI proposent la stabilisation de la situation économique et financière des entreprises, la coopération internationale dans les domaines prioritaires dans la sphère de hautes technologies de l'industrie de défense, la restructuration et la conversion de l'industrie de défense.

Dans le cadre de ces directions, une attention particulière est accordée à la création des structures compétitives intégrées, sur une base sectorielle. Les fusions horizontales et verticales ont été encouragées pour améliorer la compétitivité. La restructuration du CMI, élaborée en 1997, prévoyait la création de structures intégrées (holdings, complexes, groupes industriels) moins nombreuses et la dénationalisation, lors de la création des unions (structures intégrées), de la plupart des entreprises du secteur, qualifiées auparavant «d'importance stratégique» et "non sujettes à la privatisation», avec la transformation de ces dernières en sociétés anonymes (avec 100\% des actions en propriété d'Etat) et avec le transfert ultérieur d'actions en gérance à l'union créée. Il s'agissait aussi de réduire la liste des entreprises dont l'Etat s'était engagé à conserver $38 \%$ et $25,5 \%$ des actions, en limitant l'accès aux investisseurs étrangers et de sortir du CMI nombre de sociétés anonymes, notamment celles dont l'Etat ne dispose d'aucune action ou si la part de l'Etat peut être transférée à la compétence des fonds régionaux de la gestion patrimoine de l'Etat.

Le Gouvernement de la Fédération de Russie a pris la décision de privatiser, en 2004, 638 sociétés anonymes et 1080 entreprises fédérales unitaires d'Etat. De surcroît, une nouvelle liste des entreprises et des organisations du CMI dont la privatisation est interdite est mise en place. Sur cette liste, sont présentes 70 entreprises, en particulier CKB de la construction exacte d'appareils (Novossibirsk), CNII « Hydropribor » (Saint-Pétersbourg), CMKB «Almaz » (SaintPétersbourg) ${ }^{9}$. En même temps, un processus de création, dans le

\footnotetext{
9 Bureau Central de Conception de la construction exacte d'appareils (Novossibirsk), Institut Scientifique de Recherche Central "Hydroappareil», (SaintPétersbourg), Bureau Central de Conception Naval « Diamand » (Saint-Pétérsbourg)
(trad.).
} 
CMI, d'entreprises publiques ${ }^{10}$ fédérales (23 prévues en 2004, notamment la poudrière Lénine de Kazan) a été entrepris.

En 2001, les Bases de la politique de la Fédération de Russie dans le domaine du développement du complexe industriel de défense jusqu'à 2010 et pour la perspective ultérieure » et « le Programme d'Etat d'armement jusqu'à 2010 » ont été organisés, en vue de définir les scénarios de la stratégie générale du développement de l'économie russe à long terme. Il organise notamment les étapes de la réforme du CMI, ses buts et ses objectifs. Il s'agit principalement de diversifier la production, de créer de grandes compagnies et sociétés stables et compétitives par voie de l'intégration intersectorielle et de perfectionner le noyau de haute technologie du complexe.

Dans le CMI russe, quelques compagnies intégrées ont déjà été créées: RSK "MiG", Corporation "Armement tactique de fusées 》, Konzern PVO «Almaz-Anteï », le holding d'aviation «Sukhoï». La société « Equipement Aérospatial » est allée plus loin en signant un accord avec "Aviapribor holding ${ }^{11}$ concernant l'intégration par l'organisation d'une entre les deux compagnies, en vue d'améliorer leur compétitivité internationale ${ }^{12}$.

L'Etat a pratiquement renoncé au soutien des entreprises de défense. La position des autorités régionales à l'égard des entreprises du CMI, est quelque peu différente. Les entreprises fonctionnant sur le territoire de la région améliorent l'emploi de la population, elles atténuent l'effet du chômage, elles accroissent les recettes du budget régional et, en conséquence, elles offrent de plus grandes possibilités de solutions aux questions sociales. Lorsque l'entreprise de défense est partie prenante du tissu urbain, sa fermeture peut signifier la crise sociale et économique de la ville. La réalisation de ces décisions emprunte les voies diverses. Ainsi, pendant les réformes, les dépenses du Gouvernement de la République de Tatarstan pour le soutien des entreprises du CMI de compétence conjointe des Gouvernements de la Fédération de Russie et de la république ont représenté, en sommes cumulées, 18 milliards de dollars (au $1^{\text {er }}$ juin 2001). Dans l'oblast de

10 Entreprise publique est une entreprise gérée complétement par l'Etat, avec le recours de mécanisme de planification. Les entreprises de ce type peuvent être attachées aux autorités fédérales ou régionales, (trad.).

11 Aviaappareil bolding (trad.).

${ }^{12} \mathrm{La}$ compagnie unie réalisera le cycle complet de production sur l'ensemble des équipements de bord pour tous types d'appareils volants, militaires ou civils. Au moment de l'intégration, la corporation «Equipement Aérospatial» contrôle 55$65 \%$ du marché, et «Aviapribor holding 》 détient 10-15\% de ce marché. 
Samara, le gouverneur s'est adressé à la Douma Régionale en vue d'effacer les dettes publiques régionales accumulées par les entreprises. Le chef de la ville de Tchapaevsk, où se trouvent 5 de 8 entreprises de production de matières explosives situées dans l'oblast de Samara, a déjà promis de libérer les entreprises de leur dette de 100 millions de roubles envers le budget local.

Le soutien des entreprises par des autorités régionales s'exprime sous forme de financements budgétaires régionaux, d'allègements fiscaux et de restructuration de la dette budgétaire, mais aussi par le lobbying des intérêts des entreprises du CMI. Ainsi, par exemple, grâce au soutien du Gouvernement de la république de Carélie, l'usine de construction navale "Avant-garde », en situation financière difficile, a obtenu une licence du Ministère de la défense de la Fédération de Russie pour ${ }^{1}$ a réparation et la construction de navires militaires, qu'elle n'avait plus depuis 7 ans.

Les activités de conversion n'ont pas eu un très grand succès. Cependant, pour les entreprises d'équipement pour le secteur énergétique, les résultats, sans surprise, ont été satisfaisants, malgré l'attraction des produits importés. Le Centre d'Etat Severodvinsk de la construction de navires nucléaires est dorénavant concerné par l'exploration et l'exploitation de navires et des plates-formes du pétrole et de gaz dans les mers de Pechora et de Barents. Le programme d'équipement pour l'industrie charbonnière a obtenu aussi des résultats intéressants, mais plus modestes, dans le cadre d'un programme d'import-substitution. De nombreuses entreprises de technologie militaire nucléaire ou de missiles sont impliquées dans ce programme. Il en va de même pour la création d'un chemin de fer à haute vitesse. Cependant, l'échec de l'industrie électronique russe, qui manque singulièrement de moyens, est grave pour le Ministère de la défense. Il s'agit sans doute d'un problème crucial pour l'économie russe.

L'aide des pays occidentaux pour la conversion des industries d'armement russes a surtout servi au démantèlement des armes de destruction massive. Les programmes Nunn-Lugar a permis une assistance et un partenariat entre les firmes américaines et russes d'armement. Entre 1992 et 1994, le Congrès américain a autorisé 1,2 milliard de dollars d'investissement dans ce domaine, mais du fait du secret et des difficultés administratives, les dépenses réelles ont été réduites de moitié.

En conclusion, il y a six tendances principales au développement du CMI de Russie : 
- La transformation de la plupart des entreprises publiques du CMI en sociétés anonymes, et la vente des actions de l'Etat de certaines sociétés anonymes;

- La réduction de la liste des entreprises du CMI non sujettes à la privatisation ;

- La transformation des entreprises du CMI d'importance stratégique en entreprises publiques ;

- L'assistance aux entreprises du CMI pour la création de grandes corporations compétitives (aéronautique, industrie aérospatiale, production des substances explosives etc.) ;

- Le transfert de nombre d'entreprises du CMI sous la juridiction des autorités régionales.

- Le développement des activités civiles et la recherche de marché vers les marchés internationaux ${ }^{13}$.

\section{III. - L'industrie d'armement et la demande interne d'armes}

Les armes stratégiques constituent dorénavant le fondement même de la défense russe, étant entendu que les forces conventionnelles ont perdu leur statut et leurs financements et n'entrent plus prioritairement dans les nouveaux rapports de force.

\section{A. L'armement nucléaire}

Avec la disparition du Pacte de Varsovie, la stratégie fondée sur les forces conventionnelles n'a plus la même rationalité.

\footnotetext{
13 Aujourd'hui, sauf notables exceptions, les firmes d'armement ne produisent plus exclusivement pour l'armée. Cette transformation du comportement a été, en partie, le résultat de la baisse des budgets militaires nationaux et de l'abandon progressif des secrets technologiques qui constituaient la base de leur quasi monopole. Dans ún mouvement simultané, le secteur civil est venu vers le militaire et le militaire a dû, pour survivre, se trouver des marchés civils. Le gouvernement des Etats-Unis a entrepris des actions spécifiques pour rentabiliser les retombées technologiques militaires dans le cadre de la reconversion des industries de défense. Ainsi, le Pentagone a réduit de moitié ses fournisseurs attitrés et il a fixé des normes commerciales pour les achats militaires. Il a aussi encouragé les exportations d'armements, afin de favoriser l'émergence des économies d'échelle, de réduire les coûts unitaires, de limiter les risques financiers de la recherche-développement et d'accrốtre les effets d'apprentissage. Le prix unitaire de 500 avions est supposé 20 à $30 \%$ moindre que celui découlant de la seule production des 300 appareils nécessaires à la défense nationale.
} 
Pour Nicols Voloshin, Chef du département de développemont et de test des munitions nucléaires au ministère de l'Energie nucléaire, le complexe d'armement nucléaire russe maintient son activité et il travaille en vue de construire un bouclier nucléaire en vue de protéger l'indépendance et l'intégrité territoriale de la Russie. La recherche-développement dans l'armement nucléaire continue dans le domaine nucléaire, à Sarov (région de Nijni-Novgorod), et à Snezhinsk. Il existe aussi des bureaux de design et de recherche fondamentale autour de Moscou et de Nijni-Novgorod. Les projets concernant les SSBM continuent, mais à un très faible niveau. L'armée prolonge la vie des missiles balistiques intercontinentaux des Forces Stratégiques en vue de couvrir l'absence de nouveaux sousmarins nucléaires. C'est un instrument essentiel de la défense russe. Avec les accords et traités concernant l'interdiction des tests nucléaires, le VNIITF (Russian Nuclear Center) travaille maintenant dans des programmes écologiques avec le Mayak Production Combine, Kurchatov Institute, Khopine Radium Institute et quelques centres en Ukraine, Biélorussie et Kirghizistan. La Russie a développé des accords avec les autres pays nucléaires américains, français et chinois.

L'Agence de munitions russes, créée en 1999 a la responsabilité de la sécurité des stocks d'armes nucléaires et des armes chimiques. Cependant, en 1996, le ministère de la Défense n'a reçu au programme de destruction que $13 \%$ des ressources nécessaires, $2,2 \%$ en 1997 et $2,1 \%$ en 1998. La Douma a demandé, en juin, 2 milliards de dollars d'assistance étrangère pour détruire les armes chimiques. Au rythme actuel, selon Zinivij Pak, il faudra cent ans pour éradiquer ces armes sur le sol russe ${ }^{14}$.

Du fait des stocks existants et du retard pris dans le désarmement nucléaire, faute de moyens (quel paradoxe!), la puissance nucléaire russe reste très forte, toujours au second rang mondial loin devant les autres puissances nucléaires, hormis les Etats-Unis.

\footnotetext{
${ }^{1+}$ FAS, Weapons of Mass Destruction, WMD around the world, 3 décembre 2003.
} 
Tableau 6

Arsenal nucléaire de la Russie en 2002

\begin{tabular}{|l|c|c|c|}
\hline \multicolumn{1}{|c|}{ Type } & Création & Nombre & Ogives \\
\hline Bombardier Tu-95MS6 (Bear H-6) & 1984 & 32 & 192 \\
\hline Bombardier Tu-95MS16 (Bear H-16) & 1984 & 31 & 496 \\
\hline Bombardier Tu-160 (Blackjack) & 1987 & 15 & 180 \\
\hline ICBM. SS-16 (Satan) & 1979 & 144 & 1440 \\
\hline ICBM SS-27 & 1997 & 29 & 29 \\
\hline ICBM SS-25 (Sickle) & 1985 & 360 & 360 \\
\hline ICBM SS-24 M1 (Scalpel) & 1987 & 36 & 360 \\
\hline ICBM SS-19 (Stiletto) & 1980 & 150 & 822 \\
\hline SLBM SS-N-18 M1 (Stingway) & 1978 & 96 & 288 \\
\hline SLBM SS-N-20 (Sturgeon) & 1983 & 40 & 400 \\
\hline SLBM SS-N-23 (Skiff) & 1986 & 96 & 384 \\
\hline $\begin{array}{l}\text { Land-Based non strategic bombers and fighters, } \\
\text { Tu-22M Backfire }\end{array}$ & & 105 & 1540 \\
\hline $\begin{array}{l}\text { Naval non-strategic bombers and fighters Tu- } \\
\text { 22M Backfire }\end{array}$ & & 45 & 190 \\
\hline SLCM, SS-N-9,12, 19,21, 22 & & & 240 \\
\hline ASW Weapons, SS-N-15, 16, torpilles & & & 210 \\
\hline $\begin{array}{l}\text { Naval non strategic bombers and fighters Su-24 } \\
\text { Fencer }\end{array}$ & & 50 & 190 \\
\hline $\begin{array}{l}\text { Land-based non-strategic bombers and fighters } \\
\text { Su-24 Fencer }\end{array}$ & & 280 & 1540 \\
\hline SAM SA-5B Grammon, SA-10 Grumble & 1200 & \\
\hline
\end{tabular}

\section{B. Les industries d'armements conventionnels}

L'industrie d'armement n'est pas une activité comme une autre, elle met en cause la sécurité et la liberté. Pour des raisons purement économiques, les industriels_d'armement russes n'admettent pas la compétition sur leur territoire. Ils arguent que la production nationale est essentielle pour l'indépendance stratégique (les armes étant, en outre, parfaitement adaptées aux forces armées nationales), qu'elle participe à l'industrialisation de l'économie nationale et que le financement économise des devises. Enfin, la production d'armes développe les emplois, améliore la situation de la balance des paiements, et produit des retombées technologiques dont bénéficie le secteur civil.

Malgré les plans qui ont favorisé la réduction de la taille du complexe militaro-industriel, celui-ci reste à la fois important et parfois obsolète. Il sera difficile de doter l'armée russe de matériels performants avant une bonne décennie. Ces objectifs sont très ambitieux et difficilement acceptables socialement. En outre, les dépenses 
afférentes à ces objectifs seront très coûteuses, de l'ordre de 20 milliards de roubles, ce qui rend l'opération difficile à mettre en ceuvre. On constate que près des deux tiers des dépenses militaires vont aux forces régulières, notamment à l'armée de terre et aux forces stratégiques, si l'on n'ajoute pas la question des forces paramilitaires.

Pour les achats d'armes et la $R \& D$, la situation ne fait qu'empirer. Le renouvellement des armes se fait très mal, compte tenu des très faibles moyens du ministère de la Défense (tableaux 7 et 8).

Tableau 7

La production d'armes en Russie de 1990 à 2000

\begin{tabular}{|c|c|c|c|c|}
\hline Années & Chars & Avions légers & $\begin{array}{c}\text { Avions } \\
\text { d'attaque }\end{array}$ & Bombardiers \\
\hline 1990 & 1600 & 3400 & 400 & 40 \\
1992 & 500 & 700 & 150 & 20 \\
1994 & 40 & 400 & 50 & 2 \\
1996 & 5 & 300 & 20 & 1 \\
1998 & 10 & 250 & 30 & 0 \\
2000 & 30 & 50 & 40 & 0 \\
& & & & \\
\hline
\end{tabular}

Tableau 8

La production d'armes en Russie de 1990 à 2000 (suite)

\begin{tabular}{|c|c|c|c|}
\hline Années & $\begin{array}{c}\text { ICBMs/SLB } \\
\text { Ms }\end{array}$ & Navires de guerre majeurs & Sous-marins \\
\hline 1990 & 120 & 2 & 12 \\
1992 & 70 & 2 & 6 \\
1994 & 25 & 0 & 4 \\
1996 & 20 & 3 & 3 \\
1998 & 15 & 1 & 1 \\
2000 & 5 & 1 & 1 \\
\hline
\end{tabular}

Pour l'avenir, la production ne semble pas devoir être orientée vers la hausse (voir tableau 9, page suivante). 
Tableau 9

L'inventaire des armes majeures en Russie en 2001 et 2010

\begin{tabular}{|l|l|l|}
\hline Armes & 2000 & 2010 \\
\hline Chars modernes et véhicules blindés & 45000 & 35000 \\
\hline Artillerie & 33000 & 20000 \\
\hline Avions de combat et hélicoptères d'attaque & 2800 & 1900 \\
\hline Navires de surface et sous-marins & 95 & 60 \\
\hline
\end{tabular}

Aujourd'hui, l'Etat achète de nouveaux équipements, plus légers, moins chers. Les entreprises de chars ont quasiment abandonné cette activité ou ont connu la faillite. La moitié de la flotte d'avions a été produite avant 1985 . Seulement $1 \%$ de celle-ci a été fabriquée depuis 1995. Toutes les entreprises d'armement connaissent des difficultés économiques considérables que les subventions, souvent locales et régionales, maintiennent artificiellement en vie. Enfin, toute l'infrastructure a connu un processus de délabrement incontestable. Les deux tiers des aérodromes militaires devraient recevoir des réparations sérieuses. Sans une rapide prise de conscience, il est probable que le système sera bloqué et ne permettra pas aux troupes d'être opérationnelles. Il en va de même de la destruction des armes chimiques. Du fait de l'absence de moyens, elles sont toujours disponibles, au même titre d'ailleurs que nombre d'engins nucléaires.

La capacité de ses stocks militaires a été bien maintenue. L'obsolescence est déterminée par la demande, pas par les conditions physiques de l'actif. La valeur du stock de capital de la Russie peut passer de 50\% aujourd'hui à 90\% demain si Poutine souhaite réactiver le système de guerre soviétique. La production industrielle agrégée a diminué de 40\% pendant les années 1990, la crise dans le secteur de la défense a été deux fois plus sévère, notamment dans l'aéronautique et la production navale. La Russie produisait 870 avions par an en 1992 et 90 en 2000. Comme les armes constituaient $60 \%$ de la production industrielle, il est encore possible d'y faire appel. La Russie a en outre perdu $30 \%$ de son potentiel dans les autres Républiques aujourd'hui indépendantes.

$40 \%$ des entreprises sont proches de la faillite, notamment parce que l'Etat n'a pas rempli ses propres obligations de paiement pour des contrats déjà exécutés. Au début de l'année 1998, le gouvernement devait 18500 milliards de roubles aux entreprises de dé- 
fense $^{15}$. Dans cette situation d'arriérés permanents, le Ministère de la défense fait ce qu'il peut, pendant que les entreprises d'armement sont incapables d'assurer leur pleine production, perdant ainsi les avantages des économies d'échelle. Lorsque les contrats existent, les délais de paiement de l'Etat sont considérables, les entreprises ne paient pas leurs travailleurs et les entreprises sont en situation de faillite potentielle. Les financements importants en faveur de la conversion ont été abandonnés, mais les employés sont restés dans l'entreprise.

Le complexe militaro-industriel connait des difficultés particulières, avec des meurtres d'Igor Klimov et Sergei Shchitko (RATEP Company). Partiellement privatisé, il a plongé dans une ombre profonde du fait de ses conditions économiques catastrophiques. Il est souvent le lieu de profits illégaux et il attire les groupes de criminels organisés $^{16}$. Les industries d'armement sont de plus en plus la proie des profits illégaux). La corruption est forte (13 généraux mis en examen en 2001). La Douma estime que les pertes liées aux activités illégales dans l'armée représentent $1,5 \%$ du budget total de la défense nationale.

Les efforts du gouvernement pour régler cette situation ont été vains. Depuis que le budget de l'Etat est en léger excédent, la priorité dans ce domaine est de lutter contre l'endettement des firmes d'armement (estimé par le gouvernement russe à plus de 32 milliards de roubles), qu'il faudrait avoir réduit de moitié en janvier 2003. Or, le choix n'est pas varié. Il est souvent d'ordre binaire, soit conserver les entreprises à des fins sociales, soit les faire disparaître. Cependant, après une période de stagnation, les dépenses militaires remontent et 3 milliards de dollars a été budgété pour entreprendre une modernisation, ce qui est peu et surtout trop tard. Il existe peu d'entreprises qui financent le développement d'armes sans aide de leurs gouvernements, car ce serait trop risqué. Dans ces conditions, il ne peut pas y avoir de concurrence.

15 Sergounin A.A., Subbotin S.V., (1999), "Russian arms transfers to East Asia in the 1990s ", SIPRI Research Report $\mathrm{n}^{\circ}$ 15, Oxford University Press, New York, p. 15 16.

16 Isachenkov V., (2003), "Russian Arms Industry Under Siege », Johnson's Russia 


\section{La demande de l'armée russe}

Aujourd'hui, l'armement technologiquement au point représente seulement $20 \%$ du potentiel militaire russe. Les autres ont souvent plus de dix ans. Un tiers des matériels militaires (50\% des chars de combat et des avions de combat, $80 \%$ des hélicoptères) n'est plus en situation de combattre dans des conditions technologiques adaptées. $40 \%$ demandent de nouvelles réparations. L'effort de R\&D militaire ne dépasse pas les 15\% requis. Les achats de matériels depuis 1995 sont sporadiques et faibles. Les moyens antiaériens le 100S-300 P ne couvrent pas l'intégralité du ciel de la Russie, même si le système est supérieur à celui des Etats-Unis pour la défense elle-même et la qualité des matériels. Le système S-400 n'a toujours pas été construit. Il y a des problèmes avec la création des complexes antiaériens S-400, ce qui aurait dû être fait 4 ans plus tôt. Aucun nouveau missile n'a été créé. Les stations radars des S-400 sont testées en utilisant des missiles de S-300. Il va falloir aussi couper dans le nombre de missiles commandés. 12 satellites et fusées devaient être produits et lancés en 2004. La moitié d'entre eux pourront effectivement répondre à cette demande. Il n'a pas été produit de munitions depuis 10 ans selon Vitaly Shlyikov.

La situation est critique. L'armée a reçu 280 unités de nouvelles armes dans les trois dernières années ${ }^{17}$. On prépare un nouveau Plan 2010-2015, avec un réarmement de l'Armée. La Russie retarde ses programmes d'armement, ce qui lui supprime la parité avec le programme nucléaire. Les forces de la Navy et les forces aériennes et antiaériennes ne font l'objet d'aucune prévision claire. Les armes deviennent obsolètes, mais l'armée ne reçoit pas de nouvelles armes. La Russie avait planifié de tester le missile balistique mer Bulava en 2004, mais ces tests seront probablement retardés. La Russie ne sera pas capable de construire des sous-marins nucléaires stratégiques dans les temps ( 3 projets de sous-marins 955 et un 941 d'ici à 2010).

L'armée doit améliorer et réparer les véhicules militaires et aériens plus activement. Elle peut aussi acheter des matériaux obsolètes aux entreprises de défense. En 2003, le Ministère de la défense a été capable de vendre pour 5,8 milliards de roubles de matériels. En 2004, il espère 6,3 milliards de roubles avec les moteürs d'avion, les chars et les véhicules blindés, l'artillerie, les systèmes anti-missiles et

${ }^{17}$ Comme le Yakhont supersonic anti-ship cruise missile, le Iskander operational-tactical missile complex et de nouveaux types d'armes d'infanterie. 
les munitions. Il n'y aura pas d'achats d'avions cette année. Il s'agit plutô ${ }^{+}$de réparer et de moderniser les MiG-31, les Su-25 et les Su-27. Malgré les plans qui ont favorisé la réduction de la taille du complexe militaro-industriel, celui-ci reste à la fois important et parfois obsolète. Il sera difficile de doter l'armée russe de matériels performants avant une bonne décennie. Ces objectifs sont très ambitieux et difficilement acceptables socialement. En outre, les dépenses afférentes à ces objectifs seront très coûteuses, de l'ordre de 20 milliards de roubles, ce qui rend l'opération difficile à mettre en œuvre. On constate que près des deux tiers des dépenses militaires vont aux forces régulières, notamment à l'armée de terre et aux forces stratégiques, si l'on n'ajoute pas la question des forces paramilitaires.

\section{IV. - La question des exportations des industries d'armement}

Les exportations d'armement apparaissent comme une nouvelle nécessité pour maintenir le système d'armement en Russie. Pendant longtemps, les industries d'arment ont pensé que l'exportation était une meilleure solution que la conversion des armes. C'était une solution pour financer l'industrie d'armement. Or, le potentiel de production d'armement de la Russie excédait très largement l'importance des transferts internationaux des armes. Cette politique était éventuellement viable pour chaque entreprise, mais elle n'était pas concevable au niveau collectif. Or, les entreprises ont continué leur production sans recevoir de contributions financières en retour. Avec le système des arriérés, la volonté du gouvernement de ne pas créer des conditions d'explosion sociale, l'aide des régions pour éviter les faillites, la question des travailleurs et des factures non payées n'a pas suffisamment été prise en compte. La situation de l'industrie d'armement fin 1999 était catastrophique.

Normalement, le pays d'origine est le premier client des firmes de défense. Aux Etats-Unis, l'exportation ne compte qu'à peine pour $15 \%$ des profits des industries d'armement. Or, l'armée de la Russie n'a pas acheté de nouveaux équipements depuis près de 10 ans. Plus de $70 \%$ des recettes du CMI proviennent des exportations. Or, le coût unitaire du matériel dépend de l'échelle de production (courbes d'apprentissage, choix des processus de production, amélioration des techniques et méthodes, imputation des frais fixes sur une plus grande quantité de produits fabriqués). Faute de marchés, les armes russes 
vont devenir de plus en plus chères et de moins en moins technologiquement compétitives. Pour maintenir en vie les industries d'armement, la Russie fait appel à l'aide technique et aux exportations d'armes.

Les données sur les exportations d'armes sont très hétérogènes et elles sont sujettes à de fortes variations, selon les sources d'information. Le secret, les décalages des opérations industrielles, commerciales et financières entre la commande, la livraison, les conditions de paiement et la réalisation de la créance, ainsi que la nature particulièrement centralisée et diversifiée des accords commercialo-militaires de la France nécessitent des informations plus précises. En 2002, la Russie a exporté 4,8 milliards de dollars d'armes, mais il y a des contrôles insuffisants, notamment sur la qualité. De nombreux matériels construits pendant la période soviétique sont vendus comme neufs, avec de fortes commissions pour les intermédiaires, dans une atmosphère de grande illégalité. L'Etat cherche à reprendre le contrôle de ces revenus et de lutter contre cette corruption ambiante.

\section{A. Une nouvelle organisation}

Rosoboronexport, créée par décret en novembre 2000 par le Président Poutine, est une société d'Etat qui est l'intermédiaire dans le commerce des armes de la Russie. Elle résulte de la fusion entre deux sociétés fédérales distinctes, Rosvoorouzhenie et Promexport. Cette décision supprime une concurrence entre deux sociétés d'Etat.

Rosoboronexport ${ }^{18}$ est en charge de l'exportation de l'armement conventionnel, de la modernisation et de la réparation des armes, de la construction d'infrastructures de défense, de la promotion des produits russes et de la vente de permis de production d'armes conventionnelles. La société effectue elle-même des actions de modernisation (MiG-21, au profit de l'Inde, Mirage I et III aux moteurs russes RD-33 et missiles air-air R-73). Fin 2002, son carnet de commande a atteint 12,5 milliards de dollars. Les aéronefs de transport militaire et de combat (chasseurs Sukhoi et hélicoptères Mi) constituent $75 \%$ de ces exportations, contre $12 \%$ pour les navires, $5 \%$ pour le matériel de l'armée de terre et $3 \%$ les armes antiaériennes.

${ }^{18}$ Infoguerre.com, (2003), Focus sur Rosoboronexport : l'exportation d'armement russe, 7 Août. 
Rosoboronexport est responsable de $90 \%$ des exportations militaires russes, avec 60 pays et 35 représentations à l'étranger. La Chine, l'Inde, le Koweit, l'Egypte, l'Algérie et Chypre représentent $90 \%$ de son chiffre d'affaires. Rosoboronexport collabore avec 700 sociétés d'armement et cette société travaille étroitement avec l'Ukraine, Belarus, l'Ouzbékistan et le Kazakhstan. Elle souhaite regagner tous les marchés historiques de la Russie, par la diversification des matériels. Elle a pris le contrôle de la Russian Insurance Center (assurance) qui joue un rôle comparable à la Coface, en France. La Russie reste un exportateur important dans le secteur militaire. Il a même surpassé les Etats-Unis en 2002. Cependant, ce boom n'est que temporaire, il résulte de la vente des derniers articles de la production soviétique. Il n'a plus de complexe militaro-industriel puissant et influent en Russie. Les exportations d'armes de la Russie vont connaître une terrible récession au plus tard en $2006^{19}$. C'est le dernier souffle. Aujourd'hui, la plupart des grandes industries d'armement russes se sont converties, notamment à l'exportation d'armes, ou ont connu la faillite. La Russie' a fourni $36 \%$ des armes à l'exportation dans le monde.

\section{B. Des exportations importantes, mais en danger}

Les exportations d'armes ne répondent pas non plus aux seuls critères de la compétitivité et, compte tenu du rôle de l'Etat et de sa volonté politique d'agir sur la scène internationale, l'hypothèse selon laquelle il existerait des ventes à perte, voire paupérisantes, n'est pas dénuée de tout fondement et mériterait en tout cas d'être soigneusement analysée, à partir d'informations vérifiées et vérifiables. Les compensations'directes s'apparentent au troc. Celles-ci ne sont pas toujours favorables aux produits nationaux en concurrence avec les produits importés en échange. Les marchés sont complexes. Il est rare de recevoir du "comptant» en échange d'armes. Ainsi, la Malaisie a acheté $18 \mathrm{Su}$-30, mais elle a payé en huile de palme. Il y a aussi les transferts de technologie, les investissements sur place, la rétrocession de charges de travail, des accords de coproduction ou de soustraitance, la fabrication de composants ou accords d'assemblages sur place. D'autres dispositions intéressantes pour l'importateur. Notamment, certaines dispositions financières sont alléchantes, proches du

19 Weir F., (2003), «Russian arms exports booming: future clouded by lack of innovation », Research, Technews, Sun. June 29. 
don, avec des primes, des bonus, des réductions ou des montages financiers très avantageux.

Aujourd'hui, la Russie dispose de plus de 12,5 milliards de dollars de contrat en poche. Le Président Poutine s'en est félicité et a appelé l'Etat à aider le CMI à moderniser et à développer ses productions. Cependant, la base des consommateurs est étroite, l'innovation est faible, il existe un gaspillage des ressources et un refus des réformes qui jouent contre l'avenir. Le patrimoine militaire de l'URSS a été dilapidé. Le magasin des pièces disponibles se réduit. Aujourd'hui, les coûts de production vont croître très rapidement. Dans ce contexte, l'industrie d'armement russe va perdre sa compétitivité en termes de coûts, mais aussi en termes purement technologiques au sens militaire. En outre, les marchés traditionnels de l'Inde et de la Chine, qui ont encore besoin de l'expertise et des technologies russes, paraissent saturés, d'autant que la moitié des revenus d'exportation a porté sur le seul Sukhoi Su-30. Pour Pyadushkin, la Russie n'a pas produit un seul nouvel article depuis la fin de l'URSS.

La stratégie de développement par les exportations est très dangereuse lorsqu'elle s'applique au domaine militaire. L'exportation, nécessaire à la réduction des coûts par les économies d'échelle, crée aussi une dépendance économique. En outre, les ventes s'accompagnent de conditions de crédit particulièrement satisfaisantes pour les acheteurs. Enfin, compte tenu des exigences des acheteurs, les économies d'échelle espérées par la production en série ne sont pas très importantes, sauf pour les matériels très courants, sur lesquels peu de bénéfices sont attendus, compte tenu de la concurrence.

La lutte commerciale est importante dans le domaine des armes $^{20}$. Après des années de baisse, le marché reprend, mais les Russes n'ont plus grand chose à offrir comme nouveauté ${ }^{21}$. Lors du marché malais, les chars russes n'ont pas non plus été en succès. Le Polish T-91 a été préféré pour 64 engins. Il en va de même des missiles, alors que les moyens de défense russes sont considérés comme les meilleurs au mononde. La Malaisie préfère négocier avec le

${ }^{20}$ Kornoshchenko A., "The Russian army will soon have no weapons left ", Russia Weekly, 201, April 11, 2002).

${ }^{21} \mathrm{La}$ Russie vend toujours les mêmes armes depuis 1984. Ainsi, il a été vendu 18 MiG-29 à la Malaisie en 1994, et on essaie toujours de vendre aujourd'hui le même matériel. Les malais perdent confiance et se proposent d'acheter les F-18 américain pour leurs forces armées. 
Pakistan. Enfin, les compensations politiques ne favorisent guère l'industrie d'armement russe.

Les anciens pays de l'URSS restent des clients potentiels importants ${ }^{22}$. La Russie cherche aussi à intéresser les anciens alliés que sont le Vietnam et la Syrie, après l'échec de leur rapprochement avec l'Irak du fait de l'intervention américaine. Avec l'Iran, la question est plus difficile, même si des actions communes s'inscrivent dans le cadre d'une coalition contre l'hégémonie américaine dans la région.

\section{L'aide technique}

Les compensations impliquent une responsabilité et une action conjointe des deux partenaires dans la fabrication de certains biens exportés par l'entreprise exportatrice. Il s'agit de réduire la charge monétaire pour le pays acheteur, par la création de "rentrées » pour le pays acheteur (contre-achats différés, sous-traitance, rétrocession de charges de travail), de faire bénéficier d'apports de technologies ou des incitations pour le pays acheteur à choisir le vendeur.

Le Su-30 est un puissant appareil qui a été modernisé, mais les profits n'ont pas été réinvestis dans la $\mathrm{R} \& \mathrm{D}$. Le gouvernement indien a payé 200 millions de dollars pour moderniser son secteur aéronautique. L'Inde a obtenu le droit de produire ses propres Su$30 \mathrm{MKI}$ sous licence, un arrangement qui devrait couper cruellement les chances d'exportations futures. Il en va de même pour les chars T90 , une récente modification du T-80 soviétique. Moscou a permis à la Chine de produire le Su-27.

Aujourd'hui, la Russie aide l'armement iranien. Il s'agit de bâtir une alliance avec l'Iran et de construire un nouveau réacteur industriel nucléaire. Le succès de l'Iran dans ce secteur dépend de l'aide de la Russie. En fait, selon les officiels américains (Chris $\mathrm{La}$ Grasso), les Russes les aident aussi, au moins indirectement, à construire une arme nucléaire. Le renforcement de la coopération militaire entre la Russie et l'Iran se fait au détriment de l'industrie américaine. Les Russes ont fourni des avions, des missiles antiaériens, des sousmarins, des hélicoptères, des licences de production de véhicules blindés et de munitions. Ce marché est estimé à 4 milliards de dollars. Les Américains menacent de sanctions économiques en cas d'accords importants. L'Iran est très intéressé par la création d'un système

22 Donaldson R.H., (2002), "Domestic influences on Russian arms sales policy ",
$43^{e}$ Annual Meeting of the International Studies Association, New Orleans, Louisiana, March 24. 
antiaérien. Ceci va à l'encontre des intérêts de l'industrie américaine, qui souhaite reprendre pied dans la région. Les Etats-Unis cherchent, par tous les moyens, à décourager la Russie à agir dans ce pays.

L'industrie d'armement russe commence à développer quelques accords avec les pays industrialisés, notamment avec les EtatsUnis, sur les questions nucléaires, aéronautiques et spatiales. Cependant, ces alliances ne sont pas encore suffisamment développées.

\section{V. - Les comparaisons avec les Etats-Unis}

Avec l'effondrement du système soviétique et la disparition du Pacte de Varsovie, les cartes de la puissance mondiale ont été redistribuées. Les Etats-Unis sont devenus l'incontestable puissance hégémonique. Avec la mise en place des missiles anti-ballistiques, le gouvernement américain s'est engagé dans une recherche d'invincibilité, que le terrorisme international peut d'ailleurs partiellement contourner. Libérés de la guerre froide, les Etats-Unis se sont engagés dans la guerre économique ${ }^{23}$, puis dans la lutte contre le terrorisme. La dernière décennie met en exergue l'influence des armes sur la diplomatie américaine.

Aujourd'hui, alors même que les analystes américains parlaient encore de la supériorité de l'armée soviétique sur celles de l'OTAN en 1980, un déséquilibre considérable s'est creusé au bénéfice des EtatsUnis. Pour Brzezinski ${ }^{24}$, l'impératif technologique commande un réaménagement politique de la planète en faveur de la société américaine, définie comme la première société globale de l'histoire, modèle global de modernité sans impérialisme, aux techniques universelles. L'avenir n'est pas à l'idéologie et aux canons, mais aux réseaux. La puissance s'exprime par plusieurs caractéristiques comme la maîtrise de la finance internationale, le contrôle des marchés et de la technologie, la capacité à réduire les barrières du commerce international, la présence d'un modèle culturel dominant et exportable et la puissance militaire. On peut ajouter la puissance morale et religieuse ${ }^{25}$. En prônant un

${ }^{23}$ Daguzan J.-F, (1997), "Les Etats-Unis à la recherche de la supériorité économique », Reure Française de Géoéconomie, n², Eté.

${ }^{24}$ Brzezinski Z., (1971), La révolution technotronique, Calmann-Levv, Paris.

${ }_{25}$ Cette idée d'être du côté de Dieu a été réaffirmé par le Président Bush, après l'attaque terroriste du 11 septembre 2001. "Les attaques délibérées et meurtrières qui ont été menées hier contre notre pays étaient plus que des actes de terreur. Elles étaient des actes de guerre... La liberté et la peur, la justice et la cruauté se sont toujours fait la guerre et nous savons que, dans cette lutte, Dieu n'est pas neutre ». 
"nationalisme économique positif ${ }^{26}$ par une politique industrielle adaptée, les États-Unis se proposent de devenir le seul arbitre d'une économie désormais globalisée, par le maintien de leur hégémonie mondiale et de la direction spirituelle de la planète.

Après la guerre froide et l'effondrement du mur de Berlin, les entreprises d'armement américaines se sont rapidement restructurées, concentrées, créant de quasi-monopoles d'armes qui vont en opposition avec l'idée du marché solution de tous les problèmes. Il a même été admis par la justice américaine que, du fait de la concurrence internationale, les entreprises américaines d'armement ne peuvent se trouver en situation de monopole, ce qui leur vaut d'échapper aux lois « civiles » antitrust.

Avec la lutte anti-terroriste a conduit le gouvernement américain à reconsidérer son armement. Le secteur militaire américain doit être reconverti, au moins en partie. À l'avenir, la capacité militaire comprendra les armes de destruction massive et leurs vecteurs, les missiles de croisière, les mines sophistiquées, les installations souterraines (commandement et contrôle, stockage d'armes, voire des installations industrielles), les installations militaires situées en pleine ville, des sous-marins diesel équipés de torpilles avancées à grande vitesse, des systèmes de vision de nuit et des instruments de reconnaissance et de surveillance des cibles. L'option NMD (National Missile Defense) conduit à un recentrage sur les problèmes purement nationaux, conduisant à l'option unilatérale du «benign neglect». L'option de la sanctuarisation du territoire américain reste nationa-lement très populaire et électoralement payante. Le programme.NMD conduit au refus d'un dialogue sur cette question avec les alliés ou de négociations avec la Chine ou la Russie. Elle rejette la dissuasion en prenant comme hypothèse, non totalement dépourvue de vérité, de l'irrationalité des acteurs stratégiques. Pour l'instant, les essais d'inter-ception de missiles intercontinentaux n'ont pas encore été couronnés de succès. Cette stratégie n'est pourtant pas la seule conséquence d'un lobbying industriel, c'est une stratégie de sortie de la stratégie de la terreur (Mutual Assured Destruction, ou MAD) et de refus de celle de la dissuasion du faible au fort. C'est le retour à l'autonomie stratégique et au possible "first use». Il s'agit d'une option de capacité offensive et défensive tous azimuts.

Les Etats-Unis ont cherché à rentabiliser leurs retombées militaires dans le cadre de la reconversion des industries de défense,

${ }^{26}$ Reich R., (1993), L'économie mondialisée, Dunod, Paris. 
en réduisant d'abord de moitié les fournisseurs attitrés du Pentagone et en fixant des normes commerciales pour les achats militaires. Le complexe militaro-industriel s'est vu attribuer le leadership de secteurs industriels vitaux. Il en a profité pour introduire un nouveau système de valeurs, de nouveaux modes de fonctionnement, fondés partiellement sur l'absence de marché et l'assurance du profit. Le CMI s'internationalise, même si les marchés restent encore nationaux et si les gouvernements soutiennent encore les exportations d'armes de leurs ressortissants.

L'industrie d'armement devient ainsi tributaire du processus de globalisation. Pourtant, elle est historiquement réservée, au nom de la défense nationale, sur la question des ententes industrielles avec des entreprises étrangères. Aujourd'hui, l'internationalisation de la production et des marchés conduit à la réduction progressive des degrés de protection des entreprises nationales. Si l'industrie d'armement a souvent exercé une influence fondamentale sur la recherche-développement et plus généralement sur les technologies civiles contemporaines, ce phénomène n'est plus toujours vérifié. Les entreprises d'armement se sont restructurées, diversifiées et spécialisées. Du fait même de l'existence des forces nucléaires et de leur faible probabilité d'utilisation, d'autres stratégies sont mises en place, en complément ou en substitution partielle. En Russie, Vladimir Poutine veut redéployer son armée. Il se propose de recentrer l'appareil de défense sur quelques priorités, notainment les menaces internes, les frontières, le renforcement de l'arme nucléaire et le développement des armes de la coopération et de la diplomatie $e^{27}$.

Dans le monde, les industries d'armement de la Russie sont petites. Pour 2002 dans la zone de l'OCDE, le classement des plus grandes entreprises militaires est le suivant:

- Lockheed-Martin 22,2 milliards de dollars $(84 \%)^{28}$

- Boeing 20,8 milliards de dollars (38\%)

- Northrop-Grumman 14,2 milliards de dollars (83\%)

- Bae Systems 14,1 milliards de dollars (77\%)

- Raytheon 10,1 milliards de dollars (60\%)

- General Dynamics 9,8 milliards de dollars (71\%)

- Thales 6,2 milliards de dollars (59\%)

- EADS 5,7 milliards de dollars (20\%)

27 A ce titre, Vladimir Poutine se rapproche du concept de sécurité défini par Gorbatchev. C'est un nouveau pied de nez de l'histoire de la Russie.

${ }^{28}$ Entre parenthèses, le chiffre d'affaires militaire de la firme par rapport au chiffre d'affaires total. 
- UTC 4,6 milliards de dollars (16\%)

- Rolls-Royce 2,07 milliards de dollars (49\%).

On assiste à un mouvement de concentration considérable. En 1992, les dix premières firmes d'armement atteignaient 57,6 milliards de dollars. En 2002, elles atteignent 110 milliards de dollars, soit quasi-ment le double. En 1992, il n'y avait que deux firmes européennes, aujourd'hui elles sont quatre, avec une militarisation qui reste infé-rieure à celle des Américaines.

Dans ce contexte, l'industrie russe d'armement s'est rétrécie. Elle a connu, depuis 1991, une crise économique terrible. Selon le SIPRI, les Etats-Unis représentent aujourd'hui près de $50 \%$ des ventes mondiales d'armement, contre $3 \%$ à la Russie. Cependant, même si les dépenses militaires décroissent, cela ne devrait pas remettre en cause sa puissance militaire ${ }^{29}$. Depuis 1988, les dépenses militaires réelles de la Russie ont connu un déclin considérable. Depuis l'arrivée du Président Poutine au pouvoir, les dépenses militaires, malgré une inflation de près de $16 \%$, augmentent à nouveau. Ceci est dû partiellement à la reprise économique, qui réduit les contraintes. Pour 2002, avec des dépenses militaires de 262,9 milliards de roubles (soit à peu près 8 milliards de dollars), l'augmentation de l'effort économique de défense réelle est de l'ordre de $8 \%$, soit deux fois le montant estimé du taux de croissance de l'économie russe pour la même période. Selon le programme national d'armement, les ordres de défense devraient atteindre 188 milliards de roubles (soit, en utilisant le taux de change de décembre 2003, 6 milliards de dollars). Le gouvernement a décidé de diviser cette somme par deux. Pourtant, les ordres de défense se sont accrus de 30\% par an depuis 2001.

\section{En conclusion}

$\mathrm{La}$ « révolution dans les affaires militaires », fondée sur les développements technologiques civils, fait appel aux techniques de l'information. La RMA conduit à la montée en puissance de l'infor-mation par rapport à celle des masses de produits matériels. Il s'agit principalement de mettre en place un système des systèmes, fondé sur la numérisation, le traitement informatique et le positionnement géo-

${ }^{29}$ Weekly analysis (2003), The Russian Defense Ministry has joined a combat for the 2004 military budget, http://www.ru/chitalka/military/en/20030808.shtml, Issue $\mathrm{n}^{\circ} 90$, August. 
politique global et dans ce domaine, les Etats-Unis ont une longueur d'avance. L'économie américaine bénéficie d'une avance technologique et conceptuelle croissante dans les industries du futur (aéronautique, télécommunications, spatial, infosphère) et elle n'a cessé de renforcer son pouvoir d'influence sur les règles du jeu inter-national. La stratégie nationale d'information est une condition de la puissance,

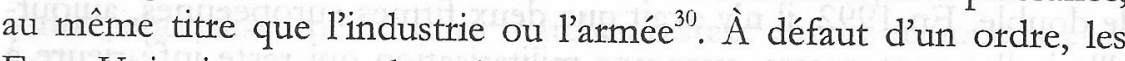
Etats-Unis imposent des règles. La loi américaine est devenue universelle, les normes techniques s'en inspirant sous l'influ-ence de leurs négociateurs.

Le «modus operandi» militaire a été transformé d'un réflexe de défense à celui, plus complexe, de peace-keeping ${ }^{31}$. La défense doit tenir compte de la lutte contre le terrorisme, de la participation au nouveau conseil Russie-OTAN et de la continuation de l'accord sur la réduction des armes nucléaires stratégiques offensives. Aujourd'hui, les efforts restent limités. Les Etats-Unis allouent $16 \%$ de leurs dépenses pour les achats nouveaux et le Pentagone souhaite arriver à $22-24 \%$. La Russie dépense 30 à 50 fois moins en R\&D que les Etats-Unis et 10 fois moins que les pays européens de l'OTAN. Si ce processus continue, la Russie cessera de mener des projets techniques dans le domaine de la défense. Aucun nouveau modèle d'armement dans les avions ou dans le transport des troupes n'est prévu. Aucun nouveau système de sécurité n'est engagé. Certaines estimations considèrent que pour rattraper le retard, il faudrait aujourd'hui 1000 milliards de dollars à l'économie russe, ce qui est irréaliste.

Aujourd'hui, le développement de la Russie n'est pas fondé sur les valeurs de la production militaire. La Russie ne présente pas les conditions fondamentales explicatives d'une course aux armements. Il n'en reste pas moins vrai que d'autres facteurs peuvent expliquer une augmentation des dépenses militaires, comme la volonté de redevenir une grande puissance stratégique. Il ne faut cependant pas oublier que les anciennes armes sont toujours présentes en Russie. Le secteur militaire est fondamentalement dépendant du niveau de développe-

\footnotetext{
${ }^{30} \mathrm{Il}$ y a quatre instruments de réflexion de l'information. D'abord, la connectivité assure aux acteurs économiques l'accès à l'information externe et interne nécessaire. De plus, les contenus, avec la concentration des efforts de production sur le renseignement économique synthétique, cherche à résoudre le trop plein d'information. Ensuite, la coordination optimise l'infrastructure nationale d'information et de «puissance cognitive ». Enfin, la sécurité assure le non effondrement des réseaux financiers, énergétiques, de communication et de transport.

31 Mayev S., (2003), Russia's armour track, http://www.ets-news.com/russia.html.
} 
ment et des capacités nationales en termes d'hommes et de capitaux en matière de recherche-développement. Les stratégies mondiales dépendent d'abord des innovations technologiques et de la diffusion des technologies avancées.

Des temps très durs attend l'armée russe dans l'année à venir. En conflit avec la Tchétchénie, elle ne pourrait supporter un autre conflit. Le retour de la superpuissance militaire de la Russie avant 2015 est fortement improbable. Si le Président Poutine souhaite la modernisation démocratique, le stock de capital s'est effondré, le complexe militaro-industriel est en ruine, la technologie a pris un grand retard, l'économie est très affaiblie, le PIB est trop petit, les dépenses militaires restent encore trop fortes, le capital fuit le pays, l'investissement étranger est insuffisant, la corruption gagne tous les secteurs de la vie sociale et le système d'éducation et de santé se détériore. Cependant, la Russie peut toujours réarmer, restaurer rapidement une puissance importante, car elle dispose d'une forte culture de mobilisation militaire, et elle sait, dans ce contexte, comment faire fonctionner efficacement ses actifs. Vitaly Shlykov a montré que les capacités industrielles russes excédaient celles des Etats-Unis pendant la guerre froide, avec upe stratégie spartiate qui a rendu le système de défense supportable. Ce système pourrait tout aussi bien revivre. Le mécanisme est endommagé, mais il est réparable

\section{Bibliographie}

Aganbeguyan, A., Fontanel, J. (1994) Un monde en transition. Les exemples de la Russie et de l'industrie d'armement. Pax Economica.

Brzezinski, Z. (1971), La révolution technotronique, Calmann Levy, Paris.

Dagusan, J-F. (1997), Les Etats-Unis à la recherche de la supériorité économique, Revue Française de Géoéconomie, ${ }^{\circ} 2$.

Donaldson, R.H. (2002), Domestic influences on Russian arms sale policy, 43th Annual Meeting of the International Studies Association, New Orleans, March 24.

FAS,(2003), Weapons of Mass Destruction, WMD around the world, 3 December.

Fontanel, J., Borissova, I. Ward, M. (1995), The principles of arms conversion in the case of Russia, Defense and Peace economics, 6(3).

Fontanel, J., Gaidar, Y. (1998), L'avenir de l'économie russe en question, PUG, Presses Universitaires de Grenoble.

Fontanel, J., Coulomb, F., Samson, I. (2001), Military conversion and transition in Russia, Pax Economica, ${ }^{\circ} 6$.

Fontanel, J., Ward, M. (2002), A hard look at the costs of peace, World Economics, 3(2).

Fontanel, J., Karlik, A. (2005), L'industrie d'armement de la Russie, Innovations (80-108).

Fontanel, J. (2002), Disarmament : A century of economic thought, Defence and Peace Economics, Tome 28, 2002. 
Fontanel, J., Samson, I. (2003), Les liaisons dangereuses entre l'Etat et l'économie russes, L'Harmattan, Paris.

Infoguerre.com (2003), Focus sur Rosoboronextport, l'exportation d'armement russe, 7 Août.

Isachenkov, V. (2003), Russian Arlms Industry Under Siege, Johnson's Russia List, 7216, June 9.

Kornoshchenko, A. (2002), The Russian army will soon have no weapons left, Russia Weekly, April, 11.

Mayev, S. (2003), Russia's armour track, http://www.etsnews.com/russia.html

Reich, R. (1993), L'économie mondialisée, Dunod, Paris.

Sergounin, A.A., Subbotin, S.V. (1999), Russian arms transfers to East Asia in the 1990s, SIPRI Resaerch Report, Oxford University Press.

Shkaratan, O., Fontanel, J. (1998), Conversion and personnel in the Russian military-industrial complex, Defense and Peace Economics 9(4).

Smith, R., Humm, A., Fontanel, J. Capital labour substitution in defence provision, in Defence Security and Development, London.

Statistichevshy bulleten (2003), Osnovnue fondy $u$ drugie nefinansovie aktivy v Rossii, ianvar.

Weekly analysis (2003) The Russian Defense Ministry has joined a combat for the 2004 military budget?, Issue $\mathrm{n}^{\circ} 90$. http://www.ru/chitalka/military/en/20030808.shtml

Weir, F. (2003), Russian arms exports booming : future cli\$ouded by lack of innovation, Research Technews, June 29.

Wines, M. (2000) Putin cuts forces by 600.000, Promising Military Overhaul, New York Times, Novembre, 10. 